\title{
Truncated Nambu-Poisson bracket and entropy formula for multiple membranes
}

\author{
Chong-Sun Chu, ${ }^{a}$ Pei-Ming Ho, ${ }^{b}$ Yutaka Matsuo $^{c}$ and Shotaro Shiba ${ }^{c}$ \\ ${ }^{a}$ Centre for Particle Theory and Department of Mathematics, University of Durham, \\ South Rd, Durham, DH1 3LE, U.K. \\ ${ }^{b}$ Department of Physics and Center for Theoretical Sciences, National Taiwan University, \\ No.1, Sec.4, Roosevelt Road, Taipei, Taiwan, R.O.C. \\ ${ }^{c}$ Department of Physics, Faculty of Science, University of Tokyo, \\ Hongo 7-3-1, Bunkyo-ku, Tokyo 113-0033, Japan \\ E-mail: chong-sun.chu@durham.ac.uk, pmho@phys.ntu.edu.tw, \\ matsuo@phys.s.u-tokyo.ac.jp, shiba@hep-th.phys.s.u-tokyo.ac.jp
}

ABSTRACT: We show that there exists a cut-off version of Nambu-Poisson bracket which defines a finite dimensional Lie 3 -algebra. The algebra still satisfies the fundamental identity and thus produces $\mathcal{N}=8$ supersymmetric BLG type equation of motion for multiple M2 branes. By counting the number of the moduli and the degree of freedom, we derive an entropy formula which scales as $N^{3 / 2}$ as expected for the multiple M2 branes.

KEYwORDS: p-branes, M-Theory. 


\section{Contents}

1. Introduction 1

2. Truncation of Nambu-Poisson bracket 2

3. Homogeneous Nambu-Poisson brackets and associated (fuzzy) geometries

4. Application to BLG model and counting entropy

5. Discussion

A. Some details on the truncated Nambu-Poisson algebra (3.16)

A.1 Structure of algebra

A.2 Invariant metric

\section{Introduction}

Bagger-Lambert-Gustavsson model [1-[] gives a prototype construction of an $\mathcal{N}=8$ superconformal field theory in 3 dimensions. The construction relies on two structures of a Lie 3-bracket: the fundamental identity which is essential to the closure of the supersymmetry transformations; and an invariant metric which allows one to convert the equation of motion to an off-shell action. The original example of BL was based on a Lie 3-algebra $A_{4}$ with a positive definite metric. In this case, one may alternatively define the theory by a Lie algebra $\mathrm{SU}(2) \times \mathrm{SU}(2)$ [5]. It was then proved that this is essentially the only possible 3 -algebra which satisfies all the requirements [6-8] (see also [9]).

It turned out that one may replace the conditions for Lie 3 -algebra by milder ones. One possibility is to permit to include a negative norm generator [10-12] (see also [13]-17]). In this case, BLG model can have gauge symmetry based on an arbitrary Lie algebra $\mathbf{g}$. Another option is to lower the supersymmetry to $\mathcal{N}=6$ where we can have $\mathrm{U}(N) \times \mathrm{U}(N)$ or $\mathrm{SU}(N) \times \mathrm{SU}(N)$ gauge symmetry [18] (see also [19-26]) which may be alternatively realized by Lie 3 -algebra which is not anti-symmetric [27].

One of the crucial test of multiple M2 brane theory is whether one can reproduce the celebrated $N^{3 / 2}$ scaling law for entropy [28] as is predicted by AdS/CFT correspondence. For any theory based on Lie algebra, however, this seems to be difficult. The number of moduli is related to the rank of the Lie algebra and the number of the generators is given by the dimension. It will produce $N^{3 / 2}$ scaling only if one consider delicately chosen tensor products of Lie groups [13] or so far hidden mechanism changes the degrees of freedom. 
In this paper, we take a different approach to this issue. Some time ago, it was proved that BLG model based on infinite dimensional Lie 3-algebra defined by Nambu-Poisson bracket is equivalent to M5 brane world volume theory [29, 30] (see also [31- 34]). What we are going to do is to cut-off this Lie 3-algebra to finite dimensions. It is actually very natural to expect to have $N^{3 / 2}$ law from the following geometrical reason.

We note that the Nambu-Poisson bracket is defined by [35],

$$
\{f, g, h\}=\sum_{\mu, \nu, \rho=1}^{3} \epsilon_{\mu \nu \rho} \partial_{\mu} f \partial_{\nu} g \partial_{\rho} h .
$$

Here $f, g, h$ are arbitrary functions of three variables $x^{1}, x^{2}, x^{3}$. Suppose we can truncate this infinite dimensional Hilbert space into a finite dimensional one, let us assume that we have $N$ degrees of freedom for each dimensions. The number of independent generators behaves as $\# G \sim N^{3}$. On the other hand, the number of M2 branes is, roughly speaking, identified with the number of the moduli which are related to mutually commuting degree of freedom. In this case, due to the structure of the Nambu-Poisson bracket, mutually commuting generators may be taken as functions which depend only on two variables, say $x^{1}, x^{2}$. The number of such generators can be estimated as $\# M \sim N^{2}$. By combining it, we have the desired scaling $\# G \sim(\# M)^{3 / 2}$ !

In this paper, by generalizing the procedure considered in [9], we show how to obtain a finite dimensional Lie 3-algebra from a truncation of the Hilbert space where NambuPoisson bracket is defined. The fundamental identity of the Lie 3-algebra is preserved by the cut-off but it becomes generally difficult to keep a non-trivial invariant metric. Therefore, although it is difficult to write BLG action, we can define the $\mathcal{N}=8$ supersymmetric equation of motion as considered in [2, 36]. The counting of the moduli is given as above and we obtain the $N^{3 / 2}$ scaling law of entropy rather robustly.

By definition, our truncated algebra becomes the infinite dimensional Lie 3-algebra from Nambu-Poisson bracket in the large $N$ limit. In this sense, it gives an intermediate geometrical structure between M2-brane and M5-brane. This is somewhat analogous to the fact that $\mathrm{D}(p+2)$-brane is obtained by collecting large $N$ limit of $\mathrm{D} p$-brane. In this sense, it may serve as a candidate of multiple M2 branes although it requires many improvements to define a realistic theory. ${ }^{1}$

For other important results on multiple M2-brane, see for example 43].

\section{Truncation of Nambu-Poisson bracket}

We start from a Nambu-Poisson bracket defined by local coordinates $x^{\mu}(\mu=1, \cdots, d)$ by

$$
\left\{f_{1}, f_{2}, f_{3}\right\}:=P\left(f_{1}, f_{2}, f_{3}\right):=\sum_{\mu_{1}, \mu_{2}, \mu_{3}=1}^{d} P^{\mu_{1} \mu_{2} \mu_{3}}(x) \partial_{\mu_{1}} f_{1} \partial_{\mu_{2}} f_{2} \partial_{\mu_{3}} f_{3}
$$

\footnotetext{
${ }^{1}$ We note that a derivation of $N^{3 / 2}$ law for M2 branes was considered previously in 37] (see also [38, 39]) in the context of Basu-Harvey equation 40] which describes a "ridge" configuration of M2-M5 system. Their analysis is based on the fuzzy $S^{3}$ defined in 41, 42]. Since it appeared before [2], the essential ingredients of the BLG model such as Lie 3-algebra and the fundamental identity were not taken into account.
} 
where $P^{\mu_{1} \mu_{2} \mu_{3}}(x)$ is an anti-symmetric tensor. In order to apply to the BLG model, it is essential to assume here that the Nambu-Poisson bracket satisfies fundamental identity,

$$
\begin{aligned}
\left\{f_{1}, f_{2},\left\{f_{3}, f_{4}, f_{5}\right\}\right\}= & \left\{\left\{f_{1}, f_{2}, f_{3}\right\}, f_{4}, f_{5}\right\} \\
& +\left\{f_{3},\left\{f_{1}, f_{2}, f_{4}\right\}, f_{5}\right\}+\left\{f_{3}, f_{4},\left\{f_{1}, f_{2}, f_{5}\right\}\right\} .
\end{aligned}
$$

The Leibniz rule,

$$
\left\{f_{0} f_{1}, f_{2}, f_{3}\right\}=f_{0}\left\{f_{1}, f_{2}, f_{3}\right\}+\left\{f_{0}, f_{2}, f_{3}\right\} f_{1},
$$

is usually required in the literature. In the context of BLG model, the role of this condition is not very clear at this moment. The fundamental identity imposes a severe constraint on $P^{\mu_{1} \mu_{2} \mu_{3}}(x)$. In mathematical literature, it is known that the fundamental identity implies the decomposability of $P$ (see for example [44] and references therein). Namely it should be rewritten as

$$
\begin{aligned}
P & =P^{\mu_{1} \mu_{2} \mu_{3}}(x) \partial_{\mu_{1}} \wedge \partial_{\mu_{2}} \wedge \partial_{\mu_{3}}=V_{1} \wedge V_{2} \wedge V_{3}, \\
V_{i}(x) & =V_{i}^{\mu}(x) \partial_{\mu} .
\end{aligned}
$$

It implies that the Nambu-Poisson bracket is essentially defined on three dimensional subspace $(\mathcal{N})$ specified by the tangent vectors $V_{i}(i=1,2,3)$. In [29, 30], it was used to obtain the M5 brane from BLG model whose world volume is the product $\mathcal{M} \times \mathcal{N}$. In the following, since we need to restrict $P^{\mu_{1} \mu_{2} \mu_{3}}(x)$ to be polynomials of fixed degree for the consistency of the cut-off, we will not use this decomposability. When $P^{\mu_{1} \mu_{2} \mu_{3}}(x)$ are homogeneous

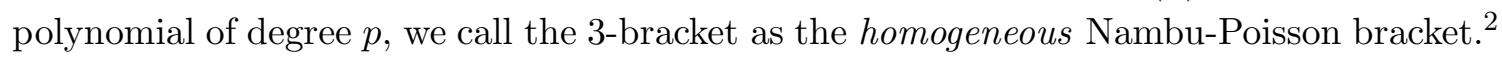

In [9], a truncation of the Nambu-Poisson bracket (2.1) which satisfies the fundamental identity was proposed. The idea was to truncate the Hilbert space $C(X)$ (functions on $X$ ) to polynomials of $x^{\mu}$ of degree $\leq N$. We will write this truncated Hilbert space as $C(X)_{N}$. For such truncation to work properly, we need to restrict the anti-symmetric tensor $P^{\mu_{1} \mu_{2} \mu_{3}}(x)$ to be a homogeneous polynomial of degree $p>0$.

\footnotetext{
${ }^{2}$ Let us briefly mention the previous studies on the quantum Nambu bracket. One of the most natural direction is to seek an analog of the Moyal product as a deformation of Poisson bracket. It was studied most extensively by Takhtajan [45] and his collaborators. Despite much efforts, however, the natural analog of the Moyal product has not been found so far. At some point, they changed the strategy and found a deformation of Nambu-Poisson bracket which was called "Zariski quantization" 46. This construction, however, needs to use an analog of the second quantized operators and is infinite dimensional by its nature. Another approach is to use a generalization of the matrix commutator (see for example 477). Although it gives rise to a very simple finite dimensional system, the triple commutator satisfies so called generalized Jacobi identity instead of the fundamental identity. In this sense, it is not obvious how to apply their algebraic structure to the BLG model. The third approach is to use the cubic matrix (three index object like " $A_{i j k}$ ") to represent the 3 -algebra (see for example [48, 49]). Although there were some success, for example in the construction of "representations" of $A_{4}$ algebra [50, the cubic matrix in general does not satisfy the fundamental identity. So it is still mysterious how to apply it to BLG model. To summarize, although there are some attractive proposals in the quantum Nambu bracket, our simple cut-off procedure of the Nambu-Poisson seems to be the first example which can be readily applicable to BLG model. We do not, of course, mean that other approaches which we mentioned are meaningless in the BLG model. On the contrary we are trying to find applications of these constructions which we hope to report in the near future.
} 
On $C(X)_{N}$, we redefine the Nambu-Poisson bracket to project out all the monomials of order $>N$. We denote such projector as $\pi_{N}$ which acts on the polynomials of $x^{\mu}$ as

$$
\begin{array}{r}
\pi_{N}\left(\sum_{n_{1}, \cdots, n_{d}=0}^{\infty} c\left(n_{1}, \cdots, n_{d}\right)\left(x^{1}\right)^{n_{1}} \cdots\left(x^{d}\right)^{n_{d}}\right) \\
=\sum_{n_{1}, \cdots, n_{d}=0}^{|\vec{n}| \leq N} c\left(n_{1}, \cdots, n_{d}\right)\left(x^{1}\right)^{n_{1}} \cdots\left(x^{d}\right)^{n_{d}},
\end{array}
$$

where $|\vec{n}|:=\sum_{i=1}^{d} n_{i}$. The Nambu-Poisson bracket on the truncated Hilbert space $C(X)_{N}$ is then defined as

$$
\left\{f_{1}, f_{2}, f_{3}\right\}_{N}:=\pi_{N}\left(P\left(f_{1}, f_{2}, f_{3}\right)\right)
$$

It satisfies the fundamental identity

$$
\begin{aligned}
\left\{f_{1}, f_{2},\left\{f_{3}, f_{4}, f_{5}\right\}_{N}\right\}_{N}= & \left\{\left\{f_{1}, f_{2}, f_{3}\right\}_{N}, f_{4}, f_{5}\right\}_{N} \\
& +\left\{f_{3},\left\{f_{1}, f_{2}, f_{4}\right\}_{N}, f_{5}\right\}_{N}+\left\{f_{3}, f_{4},\left\{f_{1}, f_{2}, f_{5}\right\}_{N}\right\}_{N},
\end{aligned}
$$

because of the following reason [9]. For simplicity, we assume $f_{i}$ to be a monomial of degree $p_{i}$. Since (2.8) is satisfied trivially if $f_{i}=$ const, one may assume $p_{i}>0$. The fundamental identity becomes nontrivial if the outer bracket is non-vanishing, namely,

$$
p_{1}+p_{2}+p_{3}+p_{4}+p_{5}-6+2 p \leq N
$$

The fundamental identity is broken if the inner bracket vanishes due to the projection. This does not happen. For example, for the left hand side of (2.8), the above inequality together with $p_{i} \geq 1$ implies

$$
p_{3}+p_{4}+p_{5} \leq N+6-2 p-p_{1}-p_{2} \leq N+4-2 p \leq N+3-p .
$$

In the last inequality, we used $p \geq 1$. Therefore whenever the outer bracket does not vanish, the value for the outer bracket is identical with the original bracket. So the FI on the truncated Hilbert space comes from the FI on the original space.

$C(X)_{N}$ is generated by finite number of monomials, $\left(x^{1}\right)^{n_{1}} \cdots\left(x^{d}\right)^{n_{d}}:=T(\vec{n})=$ $T\left(n_{1}, \cdots, n_{d}\right)$ where $n_{i} \geq 0$ and $|\vec{n}| \leq N$. The truncated Nambu-Poisson bracket defines a Lie 3-algebra,

$$
\left\{T\left(\vec{n}_{1}\right), T\left(\vec{n}_{2}\right), T\left(\vec{n}_{3}\right)\right\}_{N}=\sum_{\vec{n}_{4}} f_{\vec{n}_{4}}^{\vec{n}_{1} \vec{n}_{2} \vec{n}_{3}} T\left(\vec{n}_{4}\right)
$$

which satisfies the fundamental identity,

$$
\begin{aligned}
f^{\vec{n}_{3} \vec{n}_{4} \vec{n}_{5}} \vec{n}_{6} f^{\vec{n}_{1} \vec{n}_{2} \vec{n}_{6}} \vec{n}_{7}= & f^{\vec{n}_{1} \vec{n}_{2} \vec{n}_{3}} \vec{n}_{6} f^{\vec{n}_{6} \vec{n}_{4} \vec{n}_{5}} \vec{n}_{7} \\
& +f^{\vec{n}_{1} \vec{n}_{2} \vec{n}_{4}} \vec{n}_{6} f^{\vec{n}_{3} \vec{n}_{6} \vec{n}_{5}} \vec{n}_{7}+f^{\vec{n}_{1} \vec{n}_{2} \vec{n}_{5}} \vec{n}_{6} f^{\vec{n}_{3} \vec{n}_{4} \vec{n}_{6}} \vec{n}_{7} .
\end{aligned}
$$


We remark that the geometrical meaning of the algebra becomes clear when one takes the large $N$ limit where the algebra of polynomials can be completed in different ways and this corresponds to different topological spaces.

We note that because of the constraint $p \geq 1$, we cannot define the truncated 3 -algebra from the Jacobian,

$$
P=\partial_{1} \wedge \partial_{2} \wedge \partial_{3}
$$

As for the Leibniz rule (2.3), we have to be careful how to define the product of functions in the truncated Hilbert space. We define

$$
f \bullet \bullet_{N} g=\pi_{N}(f g),
$$

which gives a commutative and associative product on the truncated space. ${ }^{3}$ We replace the Leibniz rule by using this product rule,

$$
\left\{f_{0} \bullet_{N} f_{1}, f_{2}, f_{3}\right\}_{N}=f_{0} \bullet_{N}\left\{f_{1}, f_{2}, f_{3}\right\}_{N}+\left\{f_{0}, f_{2}, f_{3}\right\}_{N} \bullet_{N} f_{1} .
$$

We show that this condition is also satisfied for $p \geq 1$.

Let us assume that $f_{i}$ are monomials of $x$ with degree $p_{i} \geq 1$ since the Leibniz rule is trivially satisfied when $p_{0}=0$ or $p_{1}=0$. The condition that the left hand side of (2.15) is non-vanishing is

$$
p_{0}+p_{1} \leq N, \quad p_{0}+p_{1}+p_{2}+p_{3}+p-3 \leq N .
$$

Since the second condition gives a stronger condition than the first for $p \geq 1$, we take the second condition. The first term on the right hand side is non-vanishing if

$$
p_{1}+p_{2}+p_{3}+p-3 \leq N, \quad p_{0}+p_{1}+p_{2}+p_{3}+p-3 \leq N .
$$

Again the second condition gives a stronger constraint. The second term on the left hand side is non-vanishing with the same condition. To summarize, the conditions for the both sides of equation are the same. So the truncation is compatible with the Leibniz rule (2.15) for $p \geq 1$.

\section{Homogeneous Nambu-Poisson brackets and associated (fuzzy) geome- tries}

For any homogeneous Nambu-Poisson, we can define a truncated algebra for each $N$. In the following, we give some examples of homogeneous algebra which satisfies the fundamental identity and associate each algebra with a three dimensional manifold. In general, we have descriptions of the homogeneous Nambu-Poisson in terms of $d$ variables. The fact that Nambu-Poisson bracket is defined in 3-dimensions can be derived by observing that there are $d-3$ elements $f_{a}(x)$ which commute with any functions of $x$, namely,

$$
\left\{f_{a}, g, h\right\}=0, \quad \text { for any } g, h .
$$

\footnotetext{
${ }^{3}$ This reminds us of the abelian deformation of the Nambu-Poisson bracket in 46 .
} 
So one may use the hyper-surface defined by $f_{a}(x)=c_{a}(a=1, \cdots, d-3)$ as the definition of 3 dim submanifold in $\mathbf{R}^{d}$. If we introduce the cut-off, one may call the corresponding geometry as "fuzzy spaces" by employing the terminology of the noncommutative geometry although our definition of the deformation is very different.

We start from the $p=1$ case. In this case, we call the bracket as linear Nambu-Poisson bracket [51] in the following. We note that the coordinates $x^{\mu}$ define a Lie 3-subalgebra,

$$
\left\{x^{\mu_{1}}, x^{\mu_{2}}, x^{\mu_{3}}\right\}=\sum_{\mu_{4}} f_{\mu_{4}}^{\mu_{1} \mu_{2} \mu_{3}} x^{\mu_{4}}, \quad P^{\mu_{1} \mu_{2} \mu_{3}}(x)=\sum_{\mu_{4}} f_{\mu_{4}}^{\mu_{1} \mu_{2} \mu_{3}} x^{\mu_{4}} .
$$

The mathematical classification of the linear Nambu-Poisson was already made and it was reviewed in [9]. It is classified into two groups,

Type I: For each $-1 \leq r \leq 3,0 \leq s \leq \min (3-r, d-4)$ one may define the bracket as

$$
\begin{aligned}
P_{(r, s)}^{I}= & \sum_{j=1}^{r+1} \pm x^{j} \partial_{1} \wedge \cdots \partial_{j} \cdots \wedge \partial_{4} \\
& \quad+\sum_{j=1}^{s} \pm x^{n+j+1} \partial_{1} \wedge \cdots \partial_{r+j+1} \cdots \wedge \partial_{4} .
\end{aligned}
$$

Here $d$ means that we delete that element in the wedge product.

\section{Type II:}

$$
P_{a}^{I I}=\partial_{1} \wedge \partial_{2} \wedge\left(\sum_{i, j=3}^{d} a_{i j} x^{i} \partial_{j}\right) .
$$

For type I case, we can choose the plus/minus sign for each term in the summation.

In the following, we pick up interesting examples that come from this classification theorem for each $d$, the number of coordinates.

$\boldsymbol{d}=\mathbf{3 :} \quad$ The only possibility comes from the type II algebra,

$$
P=\partial_{1} \wedge \partial_{2} \wedge x^{3} \partial_{3}
$$

In this case, the $x^{3}$ may be taken as a real number or a phase $e^{i \theta_{3}}$. When $x^{3}$ is taken as real, and with an appropiate completion, the truncated algebra can be thought as a deformation of $\mathbf{R}^{3}{ }^{4}$ Due to the extra factor of $x^{3}$, the Poisson structure (3.5) breaks $O(3)$ symmetry. In the correspondence with M5 brane [29, 30], $P$ represents the 3 -form flux on M5 world volume. The breakdown of rotational symmetry comes from the fact that the 3 -form background does not respect the symmetry. When $x^{3}$ is a phase, one can think of the truncated algebra as a deformation of $\mathbf{R}^{2} \times \mathbf{S}_{+}^{1}$, where $\mathbf{S}_{+}^{1}$ is dual to the algebra of functions with only non-negative Fourier modes. In this case $P \sim \partial_{1} \wedge \partial_{2} \wedge \partial_{\theta_{3}}$ defines a Nambu-Poisson bracket on $\mathbf{R}^{2} \times \mathbf{S}_{+}^{1}$.

\footnotetext{
${ }^{4}$ To avoid possible confusion, we emphasis that this is not the standard $R^{3}$ as a Poission manifold. There the Poisson structure is $\mathrm{SO}(3)$ and translationally invariant.
} 
$\boldsymbol{d}=4$ : In this case a variety of examples come from type I. For $r=3, s=0$ case, a well known example is

$$
P=x^{1} \partial_{2} \wedge \partial_{3} \wedge \partial_{4}-x^{2} \partial_{1} \wedge \partial_{3} \wedge \partial_{4}+x^{3} \partial_{1} \wedge \partial_{2} \wedge \partial_{4}-x^{4} \partial_{1} \wedge \partial_{2} \wedge \partial_{3}
$$

In this case, the 3 -algebra generated by the coordinates is $A_{4}$. It defines a Nambu-Poisson bracket on $\mathbf{S}^{3}$ since $r^{2}=\left(x^{1}\right)^{2}+\left(x^{2}\right)^{2}+\left(x^{3}\right)^{2}+\left(x^{4}\right)^{2}$ becomes the center of the 3 -algebra. Namely,

$$
P\left(r^{2} f_{1}, f_{2}, f_{3}\right)=r^{2} P\left(f_{1}, f_{2}, f_{3}\right),
$$

for any $f_{1}, f_{2}, f_{3}$. So one may put $r^{2}=$ const. The truncated algebra defines a fuzzy $\mathbf{S}^{3}$ in $\mathbf{R}^{4}$.

From this example, by taking Wick rotation, we obtain other examples. For example, the bracket after $x^{4} \rightarrow i x^{4}$,

$$
P=x^{1} \partial_{2} \wedge \partial_{3} \wedge \partial_{4}-x^{2} \partial_{1} \wedge \partial_{3} \wedge \partial_{4}+x^{3} \partial_{1} \wedge \partial_{2} \wedge \partial_{4}+x^{4} \partial_{1} \wedge \partial_{2} \wedge \partial_{3},
$$

defines a bracket on $\mathbf{d} \mathbf{S}^{3}$ since $\left(x^{1}\right)^{2}+\left(x^{2}\right)^{2}+\left(x^{3}\right)^{2}-\left(x^{4}\right)^{2}$ becomes the center of the algebra and can be set to a constant.

Similarly after taking the Wick rotation for $x^{3}, x^{4}$, we obtain

$$
P=x^{1} \partial_{2} \wedge \partial_{3} \wedge \partial_{4}-x^{2} \partial_{1} \wedge \partial_{3} \wedge \partial_{4}-x^{3} \partial_{1} \wedge \partial_{2} \wedge \partial_{4}+x^{4} \partial_{1} \wedge \partial_{2} \wedge \partial_{3}
$$

In this case, $\left(x^{1}\right)^{2}+\left(x^{2}\right)^{2}-\left(x^{3}\right)^{2}-\left(x^{4}\right)^{2}$ becomes the center of 3 -algebra and can be set to a constant which defines $\mathbf{A d S}^{3}$.

For $r=2, s=0$, we have

$$
\begin{aligned}
P_{(2,0)} & =x^{1} \partial_{2} \wedge \partial_{3} \wedge \partial_{4}+x^{2} \partial_{1} \wedge \partial_{3} \wedge \partial_{4} \pm x^{3} \partial_{1} \wedge \partial_{2} \wedge \partial_{4} \\
& =\left(x^{1} \partial_{2} \wedge \partial_{3}+x^{2} \partial_{1} \wedge \partial_{3} \pm x^{3} \partial_{1} \wedge \partial_{2}\right) \wedge \partial_{4}
\end{aligned}
$$

The center takes the form $\left(x^{1}\right)^{2}+\left(x^{2}\right)^{2} \pm\left(x^{3}\right)^{2}$ and 3 d manifold associated with it is $\mathbf{S}^{2} \times \mathbf{R}$ or $(\mathbf{A}) \mathbf{d} \mathbf{S}^{2} \times \mathbf{R}$ where $\mathbf{R}$ is described by $x^{4}$. For finite $N$, we have a deformation of these manifold.

In order to have $s>0$, we need to take $d>4$. For example for $s=1$, we need $d=5$ and

$$
P_{2,1}=P_{2,0} \pm x^{5} \partial_{1} \wedge \partial_{2} \wedge \partial_{3} .
$$

In this case, since $x^{5}$ does not appear in the derivative, it is the center of 3 -algebra. Actually the algebra for the linear functions is identical with the Lorentzian algebra 10 -12 for $g=$ $\mathrm{SU}(2)$ or $\mathrm{SL}(2)$ where $x^{4}, x^{5}$ play the role of $T^{0}, T^{-1}$ respectively. In general the parameter $s$ represents the number of pairs of the Lorentzian generators. For smaller $r$ we can add more pair $(3-r)$ of Lorentzian generators. For $r=2, s=1$, the center of the algebra becomes

$$
\left(x^{1}\right)^{2}+\left(x^{2}\right)^{2} \pm\left(x^{3}\right)^{2} \pm 2 x^{4} x^{5}, \text { and } x^{5},
$$

to which we can assign arbitrary value. 
For $r=1$ we obtain $\mathbf{S}^{1} \times \mathbf{R}^{2}$ or $\mathbf{R}^{3}$ and its generalizations with pairs of Lorentzian generators. We note that here we obtain $\mathbf{S}^{1}$ or $\mathbf{R}^{1}$ from a constraint $\left(x^{1}\right)^{2} \pm\left(x^{2}\right)^{2}=$ const. For $r=0$, we obtain $\mathbf{R}^{3}$ with the bracket,

$$
P=x^{1} \partial_{2} \wedge \partial_{3} \wedge \partial_{4}
$$

Here $x^{1}$ becomes the center of 3-bracket and can be set to a constant.

For $r=-1$, we have only the Lorentzian pairs.

For $p>1$, we do not have the classification theorem. We have, however, a few interesting examples of Nambu-Poisson bracket where fundamental identity is satisfied.

For $p=2$, we have, for example,

$$
P=\partial_{1} \wedge x^{2} \partial_{2} \wedge x^{3} \partial_{3}
$$

If we take $x^{2,3}$ real, the we have a deformed $\mathbf{R}^{3}$ with linear flux introduced in two directions. By taking $x^{2}$ or/and $x^{3}$ to be a phase, we can also have deformed $\mathbf{R}^{2} \times \mathbf{S}_{+}^{1}$ or $\mathbf{R} \times \mathbf{T}_{+}^{2}\left(\mathbf{T}_{+}^{2}\right.$ represents $\left.\mathbf{S}_{+}^{1} \times \mathbf{S}_{+}^{1}\right)$.

Another example is

$$
P=\left(\epsilon_{\mu \nu \lambda} x^{\mu} \partial_{\nu} \wedge \partial_{\lambda}\right) \wedge x^{4} \partial_{4}
$$

which can describe deformation of $\mathbf{S}^{2} \times \mathbf{R}^{1}$ or $\mathbf{S}^{2} \times \mathbf{S}_{+}^{1}$.

For $p=3$, we have an example,

$$
P=x^{1} \partial_{1} \wedge x^{2} \partial_{2} \wedge x^{3} \partial_{3}
$$

which can describe deformed $\mathbf{R}^{3}, \mathbf{R}^{2} \times \mathbf{S}_{+}^{1}, \mathbf{R} \times \mathbf{T}_{+}^{2}$ or $\mathbf{T}_{+}^{3}$ depending on the interpretation of $x^{\mu}$.

This last example will be used in the following since it has the simplest structure. In particular, the algebra (2.11) takes the following form (after minor change of the normalization factors),

$$
\left\{T\left(\vec{n}_{1}\right), T\left(\vec{n}_{2}\right), T\left(\vec{n}_{3}\right)\right\}=\vec{n}_{1} \cdot\left(\vec{n}_{2} \times \vec{n}_{3}\right) T\left(\vec{n}_{1}+\vec{n}_{2}+\vec{n}_{3}\right) .
$$

The truncated version becomes

$$
\left\{T\left(\vec{n}_{1}\right), T\left(\vec{n}_{2}\right), T\left(\vec{n}_{3}\right)\right\}_{N}=\vec{n}_{1} \cdot\left(\vec{n}_{2} \times \vec{n}_{3}\right) \theta\left(N-\left|\sum_{i} \vec{n}_{i}\right|\right) T\left(\vec{n}_{1}+\vec{n}_{2}+\vec{n}_{3}\right)
$$

where $\left(\vec{n}_{i}\right)_{j} \geq 0$ and

$$
\theta(n)=\left\{\begin{array}{ll}
1 & n \geq 0 \\
0 & n<0
\end{array} .\right.
$$

The explicit form of the algebra for other cases is straightforward to write down. For example, $\mathbf{S}_{3}$ case eq. (3.6) is given as

$$
\left\{T\left(\vec{n}_{1}\right), T\left(\vec{n}_{2}\right), T\left(\vec{n}_{3}\right)\right\}=\epsilon_{\mu \nu \lambda \rho}\left(n_{1}\right)_{\nu}\left(n_{2}\right)_{\lambda}\left(n_{3}\right)_{\rho} T\left(\vec{n}_{1}+\vec{n}_{2}+\vec{n}_{3}-\vec{\sigma}+2 \vec{e}_{\mu}\right),
$$

where $\left(\vec{e}^{\mu}\right)_{\nu}=\delta_{\mu \nu}$ and $\vec{\sigma}=\sum_{i=1}^{4} \vec{e}_{\mu}$. The truncated 3 -algebra can be obtained by restricting the generators to $|\vec{n}| \leq N$ and including a truncation factor $\theta\left(N+2-\sum_{i}\left|\vec{n}_{i}\right|\right)$ on the right hand side. 


\section{Application to BLG model and counting entropy}

As we show in the appendix, the metric of the truncated Nambu-Poisson bracket has a trivial structure and is useless in the construction of the invariant Lagrangian. ${ }^{5}$ Nevertheless, we can write down an $\mathrm{N}=8$ supersymmetric equation of motion in terms of the structure constants of the Lie 3-algebra which satisfies the fundamental identity [2],

$$
\begin{aligned}
D^{2} X_{A}^{I}-\frac{i}{2} \bar{\Psi}_{C} \Gamma_{J}^{I} X_{D}^{J} \Psi_{B} f_{A}^{C D B}+\frac{1}{2} f_{A}^{B C D} f_{D}^{E F G} X_{B}^{J} X_{C}^{K} X_{E}^{I} X_{F}^{J} X_{G}^{K} & =0 \\
\Gamma^{\mu} D_{\mu} \Psi_{A}+\frac{1}{2} \Gamma_{I J} X_{C}^{I} X_{D}^{J} \Psi_{B} f_{A}^{C D B} & =0 \\
\left(\tilde{F}_{\mu \nu}\right)_{A}^{B}+\epsilon_{\mu \nu \lambda}\left(X_{C}^{J} D^{\lambda} X_{D}^{J}+\frac{i}{2} \bar{\Psi}_{C} \Gamma^{\lambda} \Psi_{D}\right) f_{A}^{C D B} & =0 .
\end{aligned}
$$

The SUSY transformation is

$$
\begin{aligned}
\delta X_{A}^{I} & =i \bar{\epsilon} \Gamma^{I} \Psi_{A} \\
\delta \Psi_{A} & =D_{\mu} X_{A}^{I} \Gamma^{\mu} \Gamma_{I} \epsilon-\frac{1}{6} X_{B}^{I} X_{C}^{J} X_{D}^{K} f_{A}^{B C D}{ }_{A J K} \epsilon \\
\delta\left(\tilde{A}_{\mu}\right)_{A}^{B} & =i \bar{\epsilon} \Gamma_{\mu} \Gamma_{I} X_{C}^{I} \Psi_{D} f_{A}^{C D B} .
\end{aligned}
$$

An essential point here is that the structure constant contracted with metric $f^{A B C D}=$ $f_{E}^{A B C} h^{E D}$ does not appear at all [36]. It enables us to discuss important issues such as the BPS equation or the moduli without knowing the Lagrangian.

Let us pick the algebra (3.16) and study the moduli. From the equation of motion, the moduli would be described by solutions of the equation

$$
f^{E F G}{ }_{D}^{I} X_{F}^{J} X_{G}^{K}=0 .
$$

We have to be careful in the structure of the truncated algebra. In the appendix, we show that the algebra (3.16) has a structure which is similar to the Lorentzian algebra [10-12]. Namely after removing generators which decouple from the algebra, the set of generators is classified into three subsets. If we use a notation similar to [12], (i) $\mathcal{A}_{0}^{\prime}$ : the generators which do not appear on the right hand side of 3-commutator, namely the generator $T^{D}$ where $f^{A B C}{ }_{D}=0$ for any $A, B, C$. Such generators have the form $T(\vec{k})$ where one or two components of $\vec{k}$ are zero. (ii) $\mathcal{A}_{-1}^{\prime}$ : the generators which are in the center of 3-algebra. Namely the generator $T^{A}$ where $f^{A B C}{ }_{D}=0$ for any $B, C, D$. Such generators take the form $T(\vec{k})$ where $\sum_{i} k_{i}=N-1, N$. (iii) $\hat{\mathcal{A}}$ : generators which do not belong to $\mathcal{A}_{0}^{\prime}$ nor $\mathcal{A}_{-1}^{\prime}$. The difference from [10-12] is that we have a large number $\left(O\left(N^{2}\right)\right)$ of elements in $\mathcal{A}_{0}^{\prime}$ and $\mathcal{A}_{-1}^{\prime}$.

The roles of fields in each subgroup are similar to [10-12]. Let us denote the generic fields which belong to $\mathcal{A}_{0}^{\prime}, \mathcal{A}_{-1}^{\prime}, \hat{\mathcal{A}}$ as $X, Y, Z$ respectively. Then the equation of motion

\footnotetext{
${ }^{5}$ The invariant metric has nonvanishing components only for fields which do not have interactions. This is similar to case of the algebra [36] where the metric can be derived and has the only nonvanishing component $\left\langle T^{\phi}, T^{\phi}\right\rangle \neq 0$. Of course, there may be a chance to add extra generators to obtain a nontrivial metric as in the Lorentzian 3-algebra [10-12].
} 
is written schematically as

$$
\partial^{2} X=0, \quad \partial^{2} Y=F_{1}(X, Z), \quad \partial^{2} Z=F_{2}(X, Z),
$$

and SUSY (and gauge) transformations are written similarly,

$$
\delta X=0, \quad \delta Y=G_{1}(X, Z), \quad \delta Z=G_{2}(X, Z),
$$

where $F_{1,2}, G_{1,2}$ represent some nonlinear functions. To find moduli, we can put the left hand side of equation of motion (4.8) to be zero.

First we note that there is no constraint for $Y$ from (4.7). Besides, $Y$ fields never appear in the nonlinear terms in the equations of motion. We can take any solutions of $Y$ of their equations of motion, and it will not have any effect on the rest of the fields. In this sense, the $Y$ fields should be viewed as non-physical fields, and we will not treat them as part of the moduli. ${ }^{6}$

Secondly, if we assign VEV to $X$, the field equation and the symmetry transformations do depend on the VEV. On the other hand, the SUSY (gauge) transformation (4.9) for $X$ implies that these symmetries are not violated. This behavior is what one expects for a vacuum state. On the other hand, in the Lorentzian BLG model [12], the VEV for $X_{0}^{I}$ was interpreted as the coupling constant of the super Yang-Mills theory on D2 and hence is not counted as part of the moduli space. Further analysis is needed to decide whether these are to be counted as part of the moduli space or not. However we will see that including them or not does not affect our entropy counting below.

Finally the assignment of VEV for $Z$ does not seem to have such strange behavior. Therefore, this is the degree of freedom which should be identified with the moduli of M2 brane in ordinary sense.

It turns out that the equation (4.7) can give rise to various solutions. For the 3algebra (3.16), three polynomials $f_{1}, f_{2}, f_{3}$ which depend only on two polynomials of $x$, say $g_{1}(x), g_{2}(x)$ in general commute with each other,

$$
\left\{f_{1}\left(g_{1}, g_{2}\right), f_{2}\left(g_{1}, g_{2}\right), f_{3}\left(g_{1}, g_{2}\right)\right\}_{N}=0 \text {. }
$$

Therefore the moduli space is described by (truncated) polynomials of $g_{1}(x)$ and $g_{2}(x)$. Depending on the choice of $g_{1,2}$, we have different type of "Higgs" branches.

If we take both $g_{1,2}$ as function of single variables, say $g_{1}=x^{1}, g_{2}=\left(x^{2}\right)^{m}$, all the functions of $g_{1,2}$ belong to the group $\mathcal{A}_{0}^{\prime}$. The number of such functions is of the order of $N^{2}$. As we explained above, these may or may not be counted as part of the moduli space.

On the other hand, suppose we take $g_{1,2}$ such that their polynomials depend on all the coordinates non-trivially, for example $g_{1}=x^{1}+x^{2}$ and $g_{2}=\left(x^{3}\right)^{2}$, the set of polynomials of them contains elements belonging to $\hat{\mathcal{A}}$. In this case, the VEVs are assigned to $Z$ fields and should be interpreted as the moduli of M2 branes. We can count the number of the M2 branes for given set of $g_{1,2}$. Suppose we choose them such that all the VEVs of fields

\footnotetext{
${ }^{6}$ On the other hand, if we treat them as part of the moduli, the number of solutions of (4.7) can be of order $N^{3}$. We can take 6 of the scalars $X^{I}$ to be $Y$ fields, and the rest 2 of the $X^{I}$ 's can be arbitrary. For large $N$, the number of free parameters in the 2 arbitrary fields $X^{I}$ dominates and it is proportional to $N^{3}$.
} 
can be interpreted as the moduli of M2 branes. If the degree of $g_{1,2}$ is $n_{1,2}$ respectively, the number of independent generators are approximately $\frac{N^{2}}{2 n_{1} n_{2}} \sim N^{2}$ as long as $n_{1,2}$ are much smaller than $N$. We have the estimate for the number of membrane as

$$
\# M \sim N^{2} .
$$

This permits us to calculate the behavior of the entropy. The number of fields is given as the number of generators $(\# G)$. It can be estimated as

$$
\# G=\frac{(N+1)(N+2)(N+3)}{6} \sim N^{3} / 6 \sim(\# M)^{3 / 2}
$$

This is the celebrated $N^{3 / 2}$ law for M2-brane.

One may do essentially the same counting for other $d=3$ algebras associated with $\mathbf{R}^{3}$ (3.5), (3.14) which give the same behavior. So one may guess the behavior of $N^{3 / 2}$ law as a generic feature of the $d=3$ truncated Nambu-Poisson 3-algebras.

We note that there are some subtlety if one continues to do the similar analysis for $d>3$ cases. As we have seen, there are $d-3$ generators $\phi_{s}(x)$ which satisfy,

$$
\left\{\phi_{s} f_{1}, f_{2}, f_{3}\right\}=\phi_{s}\left\{f_{1}, f_{2}, f_{3}\right\}
$$

for any $f_{1}, f_{2}, f_{3}$. One may set such generators as constant $\phi_{s}(x)=c_{s}$ and this constraints gives 3 dimensional algebra.

For the truncated algebras, since such $\phi_{s}$ has nontrivial degree as the polynomial of $x$. For example $\phi=\left(x^{1}\right)^{2}+\left(x^{2}\right)^{2}+\left(x^{3}\right)^{2}+\left(x^{4}\right)^{2}$ which appear for $\mathbf{S}^{3}$ case has degree two. So the above relation should be modified as

$$
\left\{\phi_{s} \bullet_{N} f_{1}, f_{2}, f_{3}\right\}_{N}=\phi_{s} \bullet_{N}\left\{f_{1}, f_{2}, f_{3}\right\}_{N-\left|\phi_{s}\right|}
$$

where $\left|\phi_{s}\right|$ is the degree of $\phi_{s}$. It implies that we cannot put $\phi_{s}$ to a c-number if we want to keep the fundamental identity. If we treat them as the independent generators, we would have different scaling. For example, for any $d=4$ cases, we have a simple estimate that

$$
\# M \sim O\left(N^{3}\right), \quad \# G \sim O\left(N^{4}\right) .
$$

Therefore we obtain $N^{4 / 3}$ relation between the number of membranes and the number of degree of freedom. This strange behavior for $d>3$ signals the breakdown of the truncation process which does not properly respect the local factorization of the space into 3 dimensional and $d-3$ dimensional spaces. Therefore, this anomalous scaling law should be understood as coming from an incorrect regularization of the system.

\section{Discussion}

In this paper, we proposed a series of Lie 3-algebra which has two remarkable properties,

- $N^{3 / 2}$ scaling of M2 branes with clear geometrical meaning.

- M5 brane theory in the large $N$ limit [29, 30]. 
On the other hand, it has obvious shortcomings at this moment, namely we cannot define non-trivial Lagrangian with the current form of the algebra. A hope is that one may cure it by adding some extra generators as in [10-12].

Of course, the cut-off algebra which we considered here is rather exotic algebra which was not considered seriously in the literature. For example it would be much more desirable to do similar truncation by some generalization of the Moyal product or by some generalization of the concept of matrices. We note that, however, our derivation of $N^{3 / 2}$ law is quite robust and the derivation of the scaling law will be similar even for these cases.

\section{Acknowledgments}

P.-M. H., Y. M. and S. S. appreciate partial financial support from Japan-Taiwan Joint Research Program provided by Interchange Association (Japan) by which this collaboration is made possible.

The authors thank Kazuyuki Furuuchi, Yosuke Imamura, Darren Sheng-Yu Shih, Douglas Smith, Wen-Yu Wen and Tamiaki Yoneya for helpful discussions. P.-M. H. is grateful to Anna Lee for assistance in many ways. C.-S.C. acknowledges EPSRC for an advanced research fellowship and partial support by STFC. The work of P.-M. H. is supported in part by the National Science Council, the National Center for Theoretical Sciences, and the LeCosPA Center at National Taiwan University. Y. M. is partially supported by KAKENHI (20540253).

\section{A. Some details on the truncated Nambu-Poisson algebra (3.16)}

\section{A.1 Structure of algebra}

We note that the truncated 3-algebra on (3.16) can be decomposed into three subspaces:

$\mathcal{A}_{0}$ A subspace spanned by generators $T(\vec{k})$ where one or two components of $\vec{k}$ is zero. In the definition of NP bracket, we always multiply $x_{1} x_{2} x_{3}$ after taking the derivation. So the generators which belong to $\mathcal{A}_{0}$ never appear on the right hand side of the commutator. We will denote generic generator which belongs to $\mathcal{A}_{0}$ as $T_{X}$.

$\mathcal{A}_{-1}$ A subspace spanned by generators $T(\vec{k})$ where $|\vec{k}|=N-1, N$. These generators are the center of the algebra, namely

$$
\left\{T_{Y}, T(\vec{p}), T(\vec{q})\right\}_{N}=0, \quad \text { for } \forall \vec{p}, \vec{q} \text {. }
$$

where $T_{Y}$ is a generic generator which belong to $\mathcal{A}_{-1}$. It comes the fact that we need $|\vec{p}|,|\vec{q}| \geq 1$ to have nonvanishing 3-commutator. These generators can show up on the right hand side of the 3 -bracket.

$\hat{\mathcal{A}}$ The generators which belong to neither $\mathcal{A}_{0}$ nor $\mathcal{A}_{-1}$. We will write generic elements of $\hat{\mathcal{A}}$ as $T_{Z}$. 
We note that there are some elements which belong to $\mathcal{N}=\mathcal{A}_{0} \cap \mathcal{A}_{-1}$. Since every element in this subspace has vanishing commutator with anybody else and never appears on the right hand side of the commutator, they decouple from the algebra as $T(\overrightarrow{0})$. Therefore, we have to remove them from the algebra. We will write,

$$
\mathcal{A}_{0}^{\prime}=\mathcal{A}_{0} / \mathcal{N}, \quad \mathcal{A}_{-1}^{\prime}=\mathcal{A}_{-1} / \mathcal{N},
$$

to represent the relevant part of the algebra. The number of generators which belong to each subspace is

$$
\#(\hat{\mathcal{A}}) \sim \frac{N^{3}}{6}, \quad \#\left(\mathcal{A}_{0}\right) \sim \frac{3 N^{2}}{2}, \quad \#\left(\mathcal{A}_{-1}\right) \sim N^{2}, \quad \#(\mathcal{N}) \sim 6 N .
$$

In the large $N$ limit, the number of the elements which belong to $\mathcal{A}_{0}, \mathcal{A}_{-1}$ is large $\left(O\left(N^{2}\right)\right)$ but it is still much smaller than that of $\hat{\mathcal{A}}$.

\section{A.2 Invariant metric}

For any element $T_{Y}^{a} \in \mathcal{A}_{-1}^{\prime}$ and any elements $T_{Z}^{b} \in \hat{\mathcal{A}}$, they must appear on the right hand side of 3-commutator. It implies

$$
\begin{aligned}
& \left\langle T_{Y}^{a}, T_{Y}^{b}\right\rangle=\left\langle\left[T^{P}, T^{Q}, T^{R}\right], T_{Y}^{b}\right\rangle=-\left\langle T^{R},\left[T^{P}, T^{Q}, T_{Y}^{b}\right]\right\rangle=0 \\
& \left\langle T_{Z}^{a}, T_{Y}^{b}\right\rangle=\left\langle\left[T^{P}, T^{Q}, T^{R}\right], T_{Y}^{b}\right\rangle=-\left\langle T^{R},\left[T^{P}, T^{Q}, T_{Y}^{b}\right]\right\rangle=0
\end{aligned}
$$

for some $T^{P}, T^{Q}, T^{R}$. So elements in $\mathcal{A}_{-1}^{\prime}$ must be orthogonal to any elements in $\mathcal{A}_{-1}^{\prime}$ and $\hat{\mathcal{A}}$.

Similarly, for two elements in $\mathcal{A}_{0}^{\prime}$, since they do not show up in the commutator, there are no constraint for their inner product from the symmetry:

$$
\left\langle T_{X}^{a}, T_{X}^{b}\right\rangle=K_{a b} \quad \text { (arbitrary) } .
$$

We can also deduce that any elements in $\mathcal{A}_{-1}^{\prime}$ and $\hat{\mathcal{A}}$ are orthogonal with the elements of $\mathcal{A}_{0}^{\prime}$,

$$
\left\langle T_{X}^{a}, T_{Y}^{b}\right\rangle=\left\langle T_{X}^{a}, T_{Z}^{b}\right\rangle=0
$$

A proof is as follows. For the generic elements $T_{k_{1} k_{2} k_{3}} \in \mathcal{A}_{-1}^{\prime} \cup \hat{\mathcal{A}}$, we have $k_{1}, k_{2}, k_{3} \neq 0$. So one may write it as a triple commutator,

$$
T_{k_{1} k_{2} k_{3}}=\frac{1}{k_{1} k_{2} k_{3}}\left[T_{k_{1} 00}, T_{0 k_{2} 0}, T_{00 k_{3}}\right]
$$

where $T_{k_{1} k_{2} k_{3}}:=T\left(k_{1} \vec{e}_{1}+k_{2} \vec{e}_{2}+k_{3} \vec{e}_{3}\right)$. On the other hand, any element $T_{p_{1} p_{2} p_{3}} \in \mathcal{A}_{0}^{\prime}$, one of $p_{i}$ must be zero. Let us take it $p_{1}=0$. Then we have

$$
\begin{aligned}
\left\langle T_{0 p_{2} p_{3}}, T_{k_{1} k_{2} k_{3}}\right\rangle & \propto\left\langle T_{0 p_{2} p_{3}},\left[T_{k_{1} 00}, T_{0 k_{2} 0}, T_{00 k_{3}}\right]\right\rangle \\
& =-\left\langle\left[T_{0 p_{2} p_{3}}, T_{0 k_{2} 0}, T_{00 k_{3}}\right], T_{k_{1} 00}\right\rangle=0 .
\end{aligned}
$$

Finally for any two elements $T_{p_{1} p_{2} p_{3}}, T_{q_{1} q_{2} q_{3}}$ in $\hat{\mathcal{A}}$, one can derive similarly,

$$
\begin{aligned}
\left\langle T_{p_{1} p_{2} p_{3}}, T_{q_{1} q_{2} q_{3}}\right\rangle & \propto\left\langle T_{p_{1} p_{2} p_{3}},\left[T_{q_{1} 00}, T_{0 q_{2} 0}, T_{00 q_{3}}\right]\right\rangle \\
& =-\left\langle\left[T_{p_{1} p_{2} p_{3}}, T_{0 q_{2} 0}, T_{00 q_{3}}\right], T_{q_{1} 00}\right\rangle .
\end{aligned}
$$


On the right hand side, $\left[T_{p_{1} p_{2} p_{3}}, T_{0 q_{2} 0}, T_{00 q_{3}}\right]$ is zero or belong to either $\hat{\mathcal{A}}$ or $\mathcal{A}_{-1}^{\prime}$. Since the inner product between $\hat{\mathcal{A}}$ or $\mathcal{A}_{-1}^{\prime}$ with any element in $\mathcal{A}_{0}^{\prime}$ is already shown to be zero, we arrive at

$$
\left\langle T_{Z}^{a}, T_{Z}^{b}\right\rangle=0, \quad \forall T_{Z}^{a}, T_{Z}^{b} \in \hat{\mathcal{A}}
$$

As we can see, the requirement of invariance imposes very severe constraints on the form of the metric and at the end the metric has lots of null directions, making it not useful for physical applications. The potential term of the BLG model, $\left\langle\left[X^{I}, X^{J}, X^{K}\right],\left[X^{I}, X^{J}, X^{K}\right]\right\rangle$ for example, is identically zero, because nontrivial metric components only exist for elements in $\mathcal{A}_{0}^{\prime}$, while elements in $\mathcal{A}_{0}^{\prime}$ never appear as the result of a 3 -bracket.

\section{References}

[1] J. Bagger and N. Lambert, Modeling multiple M2's, Phys. Rev. D 75 (2007) 045020 hep-th/0611108.

[2] J. Bagger and N. Lambert, Gauge symmetry and supersymmetry of multiple M2-branes, Phys. Rev. D 77 (2008) 065008 arXiv:0711.0955.

[3] J. Bagger and N. Lambert, Comments on multiple M2-branes, JHEP 02 (2008) 105 arXiv:0712.3738.

[4] A. Gustavsson, Algebraic structures on parallel M2-branes, arXiv:0709.1260.

[5] M. Van Raamsdonk, Comments on the Bagger-Lambert theory and multiple M2-branes, JHEP 05 (2008) 105 arXiv: 0803.3803.

[6] P.-A. Nagy, Prolongations of Lie algebras and applications, arXiv:0712.1398.

[7] G. Papadopoulos, M2-branes, 3-Lie algebras and Plucker relations, JHEP 05 (2008) 054 arXiv:0804.2662.

[8] J.P. Gauntlett and J.B. Gutowski, Constraining maximally supersymmetric membrane actions, arXiv:0804.3078.

[9] P.-M. Ho, R.-C. Hou and Y. Matsuo, Lie 3-algebra and multiple M2-branes, JHEP 06 (2008) 020 arXiv:0804.2110].

[10] J. Gomis, G. Milanesi and J.G. Russo, Bagger-Lambert theory for general Lie algebras, JHEP 06 (2008) 075 arXiv:0805.1012.

[11] S. Benvenuti, D. Rodriguez-Gomez, E. Tonni and H. Verlinde, $N=8$ superconformal gauge theories and M2 branes, arXiv:0805.1087.

[12] P.-M. Ho, Y. Imamura and Y. Matsuo, M2 to D2 revisited, JHEP 07 (2008) 003 arXiv:0805.1202.

[13] P. De Medeiros, J.M. Figueroa-O'Farrill and E. Mendez-Escobar, Lorentzian Lie 3-algebras and their Bagger-Lambert moduli space, JHEP 07 (2008) 111 arXiv:0805.4363.

[14] M.A. Bandres, A.E. Lipstein and J.H. Schwarz, Ghost-free superconformal action for multiple M2-branes, JHEP 07 (2008) 117 arXiv:0806.0054.

[15] J. Gomis, D. Rodriguez-Gomez, M. Van Raamsdonk and H. Verlinde, Supersymmetric Yang-Mills theory from lorentzian three-algebras, arXiv:0806.0738. 
[16] B. Ezhuthachan, S. Mukhi and C. Papageorgakis, D2 to D2, JHEP 07 (2008) 041 arXiv:0806.1639.

[17] S. Cecotti and A. Sen, Coulomb branch of the lorentzian three algebra theory, arXiv:0806.1990.

[18] O. Aharony, O. Bergman, D.L. Jafferis and J. Maldacena, $N=6$ superconformal Chern-Simons-matter theories, M2-branes and their gravity duals, arXiv:0806.1218.

[19] M. Benna, I. Klebanov, T. Klose and M. Smedback, Superconformal Chern-Simons theories and $A d S_{4} / C F T_{3}$ correspondence, arXiv:0806.1519.

[20] T. Nishioka and T. Takayanagi, On type IIA Penrose limit and $N=6$ Chern-Simons theories, JHEP 08 (2008) 001 arXiv:0806.3391.

[21] Y. Honma, S. Iso, Y. Sumitomo and S. Zhang, Scaling limit of $N=6$ superconformal Chern-Simons theories and Lorentzian Bagger-Lambert theories, arXiv:0806.3498.

[22] Y. Imamura and K. Kimura, Coulomb branch of generalized ABJM models, arXiv:0806.3727.

[23] J.A. Minahan and K. Zarembo, The Bethe ansatz for superconformal Chern-Simons, arXiv:0806.3951.

[24] D. Gaiotto, S. Giombi and X. Yin, Spin chains in $N=6$ superconformal Chern-Simons-matter theory, arXiv:0806.4589.

[25] G. Grignani, T. Harmark and M. Orselli, The $\mathrm{SU}(2) \times \mathrm{SU}(2)$ sector in the string dual of $N=6$ superconformal Chern-Simons theory, arXiv:0806.4959.

[26] G. Grignani, T. Harmark, M. Orselli and G.W. Semenoff, Finite size Giant Magnons in the string dual of $N=6$ superconformal Chern-Simons theory, arXiv:0807.0205.

[27] J. Bagger and N. Lambert, Three-algebras and $N=6$ Chern-Simons gauge theories, arXiv:0807.0163.

[28] I.R. Klebanov and A.A. Tseytlin, Entropy of near-extremal black p-branes, Nucl. Phys. B 475 (1996) 164 hep-th/9604089.

[29] P.-M. Ho and Y. Matsuo, M5 from M2, JHEP 06 (2008) 105 arXiv:0804.3629.

[30] P.-M. Ho, Y. Imamura, Y. Matsuo and S. Shiba, M5-brane in three-form flux and multiple M2-branes, JHEP 08 (2008) 014 arXiv:0805.2898.

[31] M. Li and T. Wang, M2-branes coupled to antisymmetric fluxes, JHEP 07 (2008) 093 arXiv:0805.3427.

[32] J.-H. Park and C. Sochichiu, Single M5 to multiple M2: taking off the square root of Nambu-Goto action, arXiv:0806.0335.

[33] I.A. Bandos and P.K. Townsend, Light-cone M5 and multiple M2-branes, arXiv:0806.4777.

[34] K. Furuuchi, S.-Y.D. Shih and T. Takimi, M-theory superalgebra from multiple membranes, arXiv:0806.4044.

[35] Y. Nambu, Generalized Hamiltonian dynamics, Phys. Rev. D 7 (1973) 2405.

[36] U. Gran, B.E.W. Nilsson and C. Petersson, On relating multiple M2 and D2-branes, arXiv:0804.1784. 
[37] D.S. Berman and N.B. Copland, A note on the M2-M5 brane system and fuzzy spheres, Phys. Lett. B 639 (2006) 553 hep-th/0605086.

[38] D.S. Berman, M-theory branes and their interactions, Phys. Rept. 456 (2008) 89 arXiv:0710.1707.

[39] N.B. Copland, Aspects of M-theory brane interactions and string theory symmetries, arXiv:0707.1317.

[40] A. Basu and J.A. Harvey, The M2-M5 brane system and a generalized Nahm's equation, Nucl. Phys. B 713 (2005) 136 hep-th/0412310.

[41] S. Ramgoolam, On spherical harmonics for fuzzy spheres in diverse dimensions, Nucl. Phys. B 610 (2001) 461 hep-th/0105006.

[42] S. Ramgoolam, Higher dimensional geometries related to fuzzy odd- dimensional spheres, JHEP 10 (2002) 064 hep-th/0207111.

[43] A. Gustavsson, Selfdual strings and loop space Nahm equations, JHEP 04 (2008) 083 arXiv:0802.3456;

S. Mukhi and C. Papageorgakis, M2 to D2, JHEP 05 (2008) 085 arXiv:0803.3218; M.A. Bandres, A.E. Lipstein and J.H. Schwarz, $N=8$ superconformal Chern-Simons theories, JHEP 05 (2008) 025 arXiv:0803.3242;

D.S. Berman, L.C. Tadrowski and D.C. Thompson, Aspects of multiple membranes, Nucl. Phys. B 802 (2008) 106 arXiv:0803.3611;

N. Lambert and D. Tong, Membranes on an orbifold, arXiv:0804.1114:

A. Morozov, On the problem of multiple M2 branes, JHEP 05 (2008) 076 arXiv:0804.0913;

J. Distler, S. Mukhi, C. Papageorgakis and M. Van Raamsdonk, M2-branes on M-folds, JHEP 05 (2008) 038 arXiv: 0804.1256;

J. Gomis, A.J. Salim and F. Passerini, Matrix theory of type IIB plane wave from membranes, JHEP 08 (2008) 002 arXiv:0804.2186;

E.A. Bergshoeff, M. de Roo and O. Hohm, Multiple M2-branes and the embedding tensor, Class. and Quant. Grav. 25 (2008) 142001 arXiv:0804.2201;

K. Hosomichi, K.-M. Lee and S. Lee, Mass-Deformed Bagger-Lambert theory and its BPS objects, arXiv:0804.2519;

Y. Honma, S. Iso, Y. Sumitomo and S. Zhang, Janus field theories from multiple M2 branes, Phys. Rev. D 78 (2008) 025027 arXiv:0805.1895;

H. Fuji, S. Terashima and M. Yamazaki, A new $N=4$ membrane action via orbifold, arXiv:0805.1997;

C. Krishnan and C. Maccaferri, Membranes on calibrations, JHEP 07 (2008) 005 arXiv:0805.3125;

I. Jeon, J. Kim, N. Kim, S.-W. Kim and J.-H. Park, Classification of the BPS states in Bagger-Lambert theory, JHEP 07 (2008) 056 arXiv: 0805.3236;

K. Hosomichi, K.-M. Lee, S. Lee, S. Lee and J. Park, $N=4$ superconformal Chern-Simons theories with hyper and twisted hyper multiplets, JHEP 07 (2008) 091 [arXiv:0805.3662];

S. Banerjee and A. Sen, Interpreting the M2-brane action, arXiv:0805.3930;

H. Lin, Kac-Moody extensions of 3-algebras and M2-branes, JHEP 07 (2008) 136 arXiv:0805.4003;

A. Gustavsson, One-loop corrections to Bagger-Lambert theory, arXiv:0805.4443;

F. Passerini, M2-brane superalgebra from Bagger-Lambert theory, arXiv:0806.0363;

E.A. Bergshoeff, M. de Roo, O. Hohm and D. Roest, Multiple membranes from gauged supergravity, arXiv:0806.2584; 
P. de Medeiros, J.M. Figueroa-O'Farrill and E. Mendez-Escobar, Metric Lie 3-algebras in Bagger-Lambert theory, arXiv:0806.3242;

M. Blau and M. O'Loughlin, Multiple M2-branes and plane waves, arXiv:0806.3253;

T.L. Curtright, D.B. Fairlie and C.K. Zachos, Ternary Virasoro - Witt algebra, arXiv:0806.3515;

J.M. Figueroa-O'Farrill, Metric Lie n-algebras and double extensions, arXiv:0806.3534;

J. Bedford and D. Berman, A note on quantum aspects of multiple membranes, arXiv:0806.4900;

K. Hosomichi, K.-M. Lee, S. Lee, S. Lee and J. Park, $N=5,6$ superconformal Chern-Simons theories and M2-branes on orbifolds, arXiv:0806.4977.

S. Terashima, On M5-branes in $N=6$ membrane action, arXiv:0807.0197;

S. Chakrabortty, A. Kumar and S. Jain, $w_{\infty}$ 3-algebra, arXiv:0807.0284;

S. Terashima and F. Yagi, Orbifolding the membrane action, arXiv:0807.0368.

[44] I. Vaisman, A survey on Nambu-Poisson brackets, Acta. Math. Univ. Comenianae 2 (1999) 213.

[45] L. Takhtajan, On foundation of the generalized Nambu mechanics (second version), Commun. Math. Phys. 160 (1994) 295 hep-th/9301111.

[46] G. Dito, M. Flato, D. Sternheimer and L. Takhtajan, Deformation quantization and Nambu mechanics, Commun. Math. Phys. 183 (1997) 1 hep-th/9602016.

[47] T. Curtright and C.K. Zachos, Classical and quantum Nambu mechanics, Phys. Rev. D 68 (2003) 085001 hep-th/0212267.

[48] H. Awata, M. Li, D. Minic and T. Yoneya, On the quantization of Nambu brackets, JHEP 02 (2001) 013 hep-th/9906248.

[49] Y. Kawamura, Cubic matrix, Nambu mechanics and beyond, Prog. Theor. Phys. 109 (2003) 153 hep-th/0207054.

[50] Y. Kawamura, Cubic matrix, generalized spin algebra and uncertainty relation, Prog. Theor. Phys. 110 (2003) 579 hep-th/0304149].

[51] J.-P. Dufour and N.T. Zung, Linearization of Nambu structures, Comp. Math. 117 (1999) 83 dg-ga/9707006. 